\title{
Une méthode d'approche de l'émotion dans le discours et les interactions
}

\author{
Christian Plantin ${ }^{1, *}$ \\ ${ }^{1}$ ICAR (UMR 5191), 69007 Lyon, France
}

\begin{abstract}
Résumé. Cette recherche s'appuie d'abord sur les termes structurant le champ des émotions parlées, comme affect - émotion - humeur passion - sentiment (\$2). Elle prend en compte la question du lexique riche et varié de l'émotion, termes d'émotion et termes ayant une orientation émotionnelle (§3). L'objet spécifique de la recherche est l'épisode (la séquence) émotionnelle, dont la structure interne se caractérise par ses liens spécifiques à une situation, à une action, à une transformation $d u$ corps de l'expérienceur et de ses relations aux autres participants (§4). Son étude demande qu'on articule les rôles émotionnels, expérienceur, allocateur, orchestrateur de l'émotion, à la formule élémentaire "qui éprouve quoi” (\$5).
\end{abstract}

\begin{abstract}
This research is grounded on the terms structuring the field of spoken emotions, such as affect-emotion - mood-passion-feeling (\$2). It takes into consideration the matter of the rich and diverse vocabulary of emotion, terms of emotion and terms having an emotional orientation (\$3). The focus of the research is the emotion episode (sequence), whose internal structure is marked by its specific ties with a situation, an action, a transformation of the body of the experiencer and of his relationships with the other participants $(\$ 4)$. In order to study the emotion episode, this contribution articulates the emotional roles, emotion experiencer, allocator, orchestrator, to the basic formula "who feels what" (§5).
\end{abstract}

\section{Introduction : Construire des représentations}

L'étude linguistique de l'émotion peut s'effectuer à différents niveaux, selon qu'elle touche, par exemple, au lexique de l'émotion, à la syntaxe ou à la phonétique des énoncés d'émotions, à l'organisation textuelle-interactionnelle des épisodes émotionnels. Cette étude propose une méthodologie permettant de construire des représentations de ce dernier niveau, celui de la séquence émotionnelle. Une représentation est une description analytique de données correctement délimitées, corpus ou échantillons, prélevées sur un flux de langage.

Une méthodologie peut être évaluée selon la qualité des représentations qu'elle permet de construire. La représentation doit d'abord être descriptivement adéquate, et rendre

*christian.plantin@,univ-lyon2.fr 
compte des données, sur la base de l'état de l'art des études linguistiques. Elle ne doit pas heurter l'intuition analytique des locuteurs informés non spécialistes; si elle le fait, elle doit explicitement en donner les raisons. Une bonne représentation doit en outre être explicative, c'est-à-dire éclairer et enrichir les données considérées; par exemple, dans le cas de l'émotion, en explicitant la cohérence entre différents niveaux et types d'expression des émotions. On pourrait également considérer un critère d'applicabilité ou de flexibilité, qui considère la capacité de la méthodologie à fournir des résultats intéressants pour les nombreuses autres disciplines qui s'intéressent aux émotions.

Les études linguistiques fournissent les ressources conceptuelles et méthodologiques nécessaires à la construction de représentations. On trouve de précieuses ressources complémentaires notamment en psychologie, en philosophie et dans toutes les disciplines s'intéressant aux émotions. Elles fournissent en particulier les bases permettant d'enrégimenter quelque peu les termes couvrant le champ de l'émotion, c'est-à-dire de les définir et de les organiser de façon à les rendre exploitables dans le cadre de l'étude proposée $(\$ 2)$. Le travail de repérage du lexique de l'émotion prend en compte non seulement les termes d'émotions, dont il est possible d'établir une liste substantielle, mais aussi les termes ayant une orientation émotionnelle (\$3). L'objet spécifique de la recherche est l'épisode (la séquence) émotionnelle, dont la structure interne se caractérise par ses liens spécifiques à une situation et à une action, c'est-à-dire une transformation du corps de l'expérienceur et de ses relations aux autres participants (\$4). Son étude demande qu'on articule les rôles émotionnels, expérienceur, allocateur, orchestrateur de l'émotion, à partir de la formule élémentaire "qui éprouve quoi" (\$5). Pour des raisons d'espace, nous ne traiterons pas les questions relatives à la situation source de l'émotion, ni celles de la sémiotique corporelle de l'émotion.

\section{Termes couvrants : Affect - Émotion - Éprouvé - Humeur - Passion - Pathos - Sentiment - Tempérament}

Les termes génériques, au sens strict, désignent des genres réunissant différentes espèces réunies sur la base de leurs traits communs, chacune de ces espèces se caractérisant par une propriété spécifique, sa différence. Les taxonomies naturelles sont le meilleur exemple de telles organisations. Par exemple, chien est un terme générique regroupant les espèces désignées par les termes caniche, Labrador, etc. En première approximation, la peur, la colère, la satisfaction... sont des espèces attachées au genre émotion; peur, colère, satisfaction... sont des hyponymes de l'hyperonyme émotion.

On peut utiliser l'expression terme couvrant pour désigner l'un quelconque d'un ensemble de termes généraux, renvoyant globalement au champ de l'émotion : affect émotion - éprouvé - humeur - passion - pathos - sentiment. L'usage quotidien de ces termes est assez flou, et leurs significations peuvent se recouvrir. Les considérations suivantes peuvent aider à les caractériser, dans leurs dimensions sémantiques, historiques et culturelles.

Ces termes sont marqués par leur discipline d'origine et par l'histoire. Ils renvoient au champ des émotions vu sous l'angle d'une discipline, d'une époque, d'une sensibilité. Affect est lié à la psychanalyse ; émotion, à la psychologie ; humeur, à la médecine ; éprouvé à la sémiotique; pathos à la rhétorique. Passion et sentiment sont liés à des périodes historiques différentes, passion à la philosophie morale, à la peinture et à la littérature classiques $\left(17^{\mathrm{e}}-\right.$ $18^{\mathrm{e}}$ siècle) [1] ; sentiment à la période romantique $\left(18^{\mathrm{e}}-19^{\mathrm{e}}\right.$ siècle $)$ [2].

Ces termes couvrants ont également des orientations sémantiques différentes. Ils peuvent s'analyser selon les poids respectifs des différentes composantes associées au syndrome émotionnel en général (voir §4.3), c'est-à-dire l'accent qu'ils mettent plus ou moins $(+/-)$ sur : 
- la conscience que l'expérienceur peut avoir de son émotion : affect et éprouvé sont () de ce point de vue, par rapport à sentiment ou émotion $(+)$;

— le contenu physiologique associé : humeur est $(+)$ de ce point de vue, par rapport à sentiment $(-)$;

- le type de cognition associé, qui est différent pour sentiment (cognition consciente) et pour émotion (cognition perceptuelle) ;

- le type de contrôle associé : contrôle social pour émotion, contrôle éthique et religieux pour passion.

Il est difficile de conceptualiser ces termes, en attachant à chacun d'eux un sens stable et clairement différencié. Néanmoins, ils sont indispensables à la description du champ linguistique de l'émotion; nous en tiendrons aux perspectives suivantes (d'après [3]). Émotion, humeur [mood], tempérament, sentiment, passion sont définis en relation avec trois dimensions: durée; conscience; source.

Émotions et affects sont à la base du vécu émotionnel. Les émotions sont conscientes ; elles se développent et se gèrent en relation avec une source qui peut être très précise. Les affects et les sensations (feelings) correspondent à des zones émotionnelles diffuses ; leur source est moins clairement élucidable que celle des émotions.

Tempérament et humeur (mood) définissent le niveau des dispositions. L'humeur (bon / mauvais poil) correspond à une orientation émotionnelle locale, moins consciente que l'émotion, dont elle forme le substrat ; la source de l'humeur peut être interne ou externe ; sa durée est plus longue que celle des (micro-)émotions. Le tempérament (peureux, colérique) correspond à une orientation émotionnelle permanente (prédisposition) caractérisant une personne.

Dans ce cadre de référence, un sentiment est une cognition élaborée en relation avec un système d'émotions de façon à déterminer une attitude. Une passion se définit en relation avec un objet précis et exclusif : football, timbres-poste, autre personne. La passion est une disposition permanente à produire activement des occasions d'intense émotion.

\section{Termes d'émotion : disciplines et listes}

On trouve des listes de termes d'émotion en rhétorique, philosophie, théologie, psychologie, linguistique, d'où une première définition: dans notre culture, un terme d'émotion est un mot figurant dans une liste de termes d'émotions ; un tel terme correspond à une description d'une émotion typique. La première de ces listes est celle d'Aristote dans

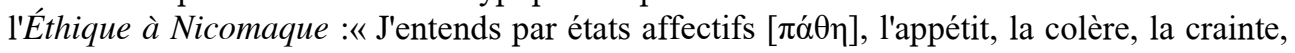
l'audace, l'envie, la joie, l'amitié, la haine, le regret de ce qui a plu, la jalousie, la pitié, bref toutes les inclinations accompagnées de plaisir et de peine » [4, p. 101].

"États affectifs" traduit le mot grec pathe [ $\pi \dot{\alpha} \theta \eta]$. Les traductions anglaises traduisent par "passion" ou "emotion". Aristote donne d'abord une définition en extension, par énumération des espèces, puis une définition par intension, précisant le genre (inclinations), puis la différence spécifique des émotions (être accompagnées de plaisir ou de peine).

La liste G\&S [5] a le mérite d'énumérer un nombre respectable de termes d'émotion, cent quarante-trois. En pratique, pour savoir si un terme est un terme d'émotion on regarde d'abord s'il figure ou non dans cette liste de termes, qui ont été extraits du dictionnaire $L e$ Petit Robert sur la base des critères suivants :

A) Les termes doivent se référer à des conditions intérieures et mentales.

B) Ils doivent impliquer un état mental transitoire.

C) Ils doivent se rapporter à des aspects affectifs, bien qu'ils puissent aussi se référer à la connaissance, au comportement, ou aux modifications physiologiques et expressives qui accompagnent les émotions. [5, p. 79] 
Cette liste, comme celle des psychologues et des philosophes, est uniquement composée de substantifs. Les critères font écho aux définitions de l'émotion proposées par les psychologues pour l'émotion comme syndrome (voir $\$ 4.3 .1$ ), touchant l'état affectif, l'état cognitif, le comportement et les productions vocales, mimiques, posturales et gestuelles (VMPG).

\begin{tabular}{|c|c|}
\hline 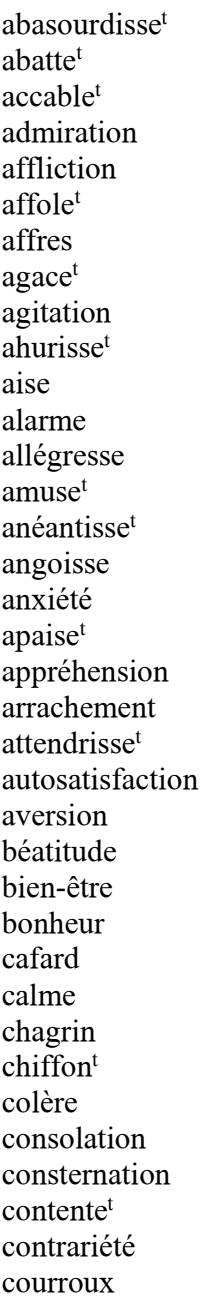 & 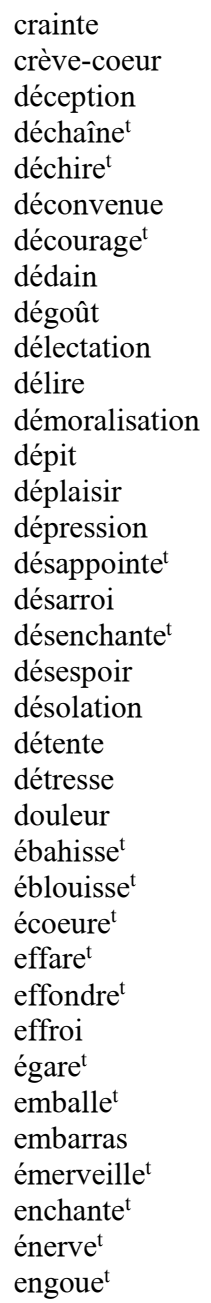 \\
\hline
\end{tabular}

ennui
enthousiasme
épatement
épouvante
étonne
euphorie
exaltation
exaspération
excitation
extase
exultation
frayeur
frustration
fureur
furie
gaieté
gène
griserie
hargne
hébéte
hilarité
horreur
humiliation
indignation
inquiétude
insatisfaction
irritation
ivresse
joie
jubilation
langueur
lassitude
liesse
malaise
mécontente
mélancolie

mépris
nervosité
oppression
paix
panique
peine
peur
plaisir
prostration
quiétude
rage
ravisse
réjouissance
répulsion
satisfaction
sérénité
souffrance
soulage
spleen
stupéfaction
stupeur
surexcitation
surprise
tension
terreur
tourment
trac
tranquillité
transe
tremble
triomphe
tristesse
trouble
vague à l'âme
vertige

\subsection{Extension sémantique de la liste : synonymes et antonymes}

Cette liste constitue une référence provisoire, dans la mesure où elle peut être considérablement étendue par les moyens suivants.

- Par intégration de toute la famille morpho-lexicale sémantiquement homogène à laquelle appartient le substantif figurant dans la liste. Les sept premiers termes de la liste G\&S se développent comme suit:

abasourdissement : abasourdir, abasourdi, abasourdissant

abattement : abattre, abattu

accablement : accabler, accablé, accablant

admiration : admirer, admirable, admiratif, admirablement

affliction : affliger, affligé, afflictif

affolement : affoler, affolé, affolant 
affres: n'a pas de développement morpho-lexical

- Par intégration des termes dits synonymes et antonymes. Par exemple, selon [6], abasourdi admet les synonymes ou parasynonymes suivants :

Abasourdi : abêti - ébahi - éberlué - ébloui - abruti - ahuri - assommé - étonné - étourdi - évaporé - consterné - distrait - effaré - hébété - rêveur - stupéfait

Abasourdi enrichit donc la liste G\&S des 8 termes suivants : abêti-éberlué - abruti assommé - étourdi - évaporé - distrait - rêveur.

- Par intégration des termes impactés par émotion et/ou par un terme d'émotion

Si le mot $[m]$ est utilisé dans la définition des mots $a, b, c \ldots$ nous dirons que $[m]$ impacte les mots $a, b, c \ldots$ et que les mots $a, b, c \ldots$ sont impactés par le mot $[m]$. Les mots sont définis selon un certain nombre d'acceptions. Par convention, nous considérerons qu'un mot est impacté dès qu'une de ses acceptions est impactée.

Par exemple, dans le TLFi le mot colère impacte le mot tomate [7] :

B. P. compar. ou p. anal.

1. a) [P. compar. avec la couleur de la tomate] Etre rouge comme une tomate. < Avoir les joues, le visage rouge de honte, de timidité, de confusion, de colère.> Elle me prit la main, la serra (...) et avec un soupir demi-pâmé, ces soupirs qui viennent d'en bas, elle me dit: "Oh! ne me regardez pas comme ça, mon enfant. » Je devins plus rouge qu'une tomate et plus timide encore que d'habitude, naturellement (MAUPASS., Contes et nouv., t. 1, Verrou, 1882, p. 816).

Les chevrons délimitent la définition proprement dite ${ }^{\dagger}$, qui contient le mot colère.

Le domaine lexicographique associé à $[m]$, noté $\mathrm{D}[\mathrm{m}]$, est défini comme l'ensemble des mots $a, b, c \ldots$ impactés par ce mot $[m]$. La réunion des domaines lexicographiques associés à un mot dans différents dictionnaires donne une idée du domaine lexical couvert par ce mot. Dans le $T L F i$, le domaine lexicographique de colère comprend 169 termes, voir liste dans [8].

Le mot impactant $[m]$ appartient généralement à une Famille Morpho-Lexicale Sémantiquement Homogène (Famille ML-SH), abrégé en “famille”, s'il n'y a pas de risque d'ambiguïté.

$$
[M]=[m 1, m 2, m 3, \ldots]
$$

On note $\left[M^{*}\right]$ la famille ML-SH du mot $[m]$. La famille ML-SH de émotion, notée $\left[E^{E} M O T I O N^{*}\right]^{\ddagger}$, est constituée des mots suivants :

[émoi, émotion, émotif, émotivité, émotionnel, émouvoir, émouvant, émotionner, émotionnant]

L'homogénéité sémantique de cette famille n'est pas forcément parfaite ; intuitivement, émouvoir quelqu'un c'est plutôt produire en lui une émotion tendre, alors qu'une réaction émotionnelle peut être de l'indignation ou de la colère. Autrement dit, les impacts de émouvoir sont a priori plus spécifiques que ceux de émotion, mais ils restent liés à émotion.

Dans la définition d'un autre mot, le mot impactant apparaît sous une certaine morphologie, selon sa catégorie grammaticale. Par exemple, les impacts de [émotion], $\mathrm{D}$ [émotion] sont la somme des impacts de émotion et de émotions; $\mathrm{D}$ [émotif] réunit les impacts de émotif, émotive, émotifs, émotives; $\mathrm{D}$ [émouvoir] l'ensemble des impacts des formes conjuguées de ce verbe.

$\mathrm{D}\left[M^{*}\right]$ est le domaine lexicographique de la famille $\left[M^{*}\right]$. Tous les mots impactés par un membre de la famille [ÉMOTION*] appartiennent au domaine lexical recherché, noté

\footnotetext{
$\dagger$ Nous appelons ici "définition" le segment sélectionné par la recherche automatique. Ce segment correspond ici à une énumération d'émotions associées à une transformation de la couleur du visage. * Pour éviter des notations cabalistiques de racines ou de bases lexicales, les familles ML-SH seront notées par le mot complet le plus impactant, accompagné d'un astérisque $<*>$.
} 
$\mathrm{D}\left[E^{E} M O T I O N^{*}\right]$. Si nécessaire, on peut mentionner le dictionnaire sur lequel s'appuie la recherche : D[ÉMOTION* $]_{[\text {Dictionnaire }]}$ — par ex. D[ÉMOTION* $]_{[T L F i]}$, voir [9].

Le domaine lexicographique ainsi obtenu à partir d'un terme d'émotion se structure en fonction du scénario de l'émotion en question, voir [8] pour colère, [9] pour émotion.

Ces résultats sont établis de façon indépendante des intuitions de l'analyste. Ils montrent que le nombre de termes d'émotions et de termes ayant une orientation émotionnelle est sans commune mesure avec les listes courantes d'émotions dites "de base", qui couvrent, dans le meilleur des cas, quelques dizaines de termes.

La méthode et les notions présentées ci-dessus sont indépendantes de la question des émotions. On pourrait les utiliser pour définir les primitifs lexicographiques d'une langue pour un dictionnaire, c'est-à-dire l'ensemble des mots impactants (définisseurs et définis), opposé à l'ensemble des mots seulement impactés (définis, jamais définisseurs).

Cette méthode permettrait également de comparer, d'une part, l'ensemble de mots associés à un mot donné dans l'usage tel qu'on l'établit par les méthodes lexicométriques (voir par exemple [10]), et, d'autre part, l'ensemble de mots impactés par ce mot dans un dictionnaire, qui se propose, entre autres choses, d'exprimer cet usage.

\section{L'émotion, syndrome et flux}

\section{1 Émotion émergente, émotion persistante}

Émerger a deux emplois distincts. Émerger ${ }_{1}$ signifie «sortir d'un liquide» ou d'un milieu homogène (TLFi, Émerger), par un mouvement ascendant. Émerger ${ }_{1}$ fait référence au début du processus qui conduira à émerger 2 «être hors d'un liquide » ou d'un milieu homogène quelconque. Émerger ${ }_{1}$ est inchoatif et émerger ${ }_{2}$ résultatif.

Les émotions émergentes ${ }_{1}$ sont des émotions in statu nascendi; elles apparaissent et se développent dans le flux de l'interaction. On peut appeler émotions persistantes les émotions émergées ${ }_{2}$, telles qu'elles se développent par-delà leur séquence émergente ${ }_{1}$. Les émotions évoquées, partagées et racontées sont des émotions persistantes, bien connues de leur expérienceur. On pourrait les appeler re-émotions : rappelées et revécues.

\subsection{Macro-émotion, micro-émotion}

On peut opposer des émotions longues, mémorables (macro-émotions, grandes émotions, émotions fortes) et émotions courtes (micro-émotions, petites émotions [11]). Les macroémotions sont des émotions persistantes; la durée de vie des micro-émotions est limitée à leur séquence émergente.

\subsection{1 Émotions longues}

L'émotion longue est caractérisée par les traits suivants [9] : (i) l'émotion est intense ; (ii) l'événement associé est exceptionnel (en référence aux intérêts et aux valeurs de l'expérienceur); (iii) l'émotion est thématisée ; (iv) elle est mémorisée dans la mémoire longue ; (v) elle est socialisée, racontée ; (vi) sa gestion est longue et complexe, et implique plusieurs types d'action; elle s'effectue en discontinu, au cours de différents épisodes ; (vii) elle est restructurante, en ce qu'elle altère le niveau thymique de l'expérienceur (il ne récupère pas forcément son état antérieur). Plusieurs exemples sont présentés dans [12].

\subsection{2 Émotions courtes}


L'émotion typiquement associée aux interjections dans la vie courante est une microémotion, comme dans le cas suivant. Pour l'analyse détaillée de cet extrait, voir [13].

\section{Corpus : Conversations familières - visites =>> Transcription : Clodif Q4}

Retrouver directement cette attestation avec le code : 79/86

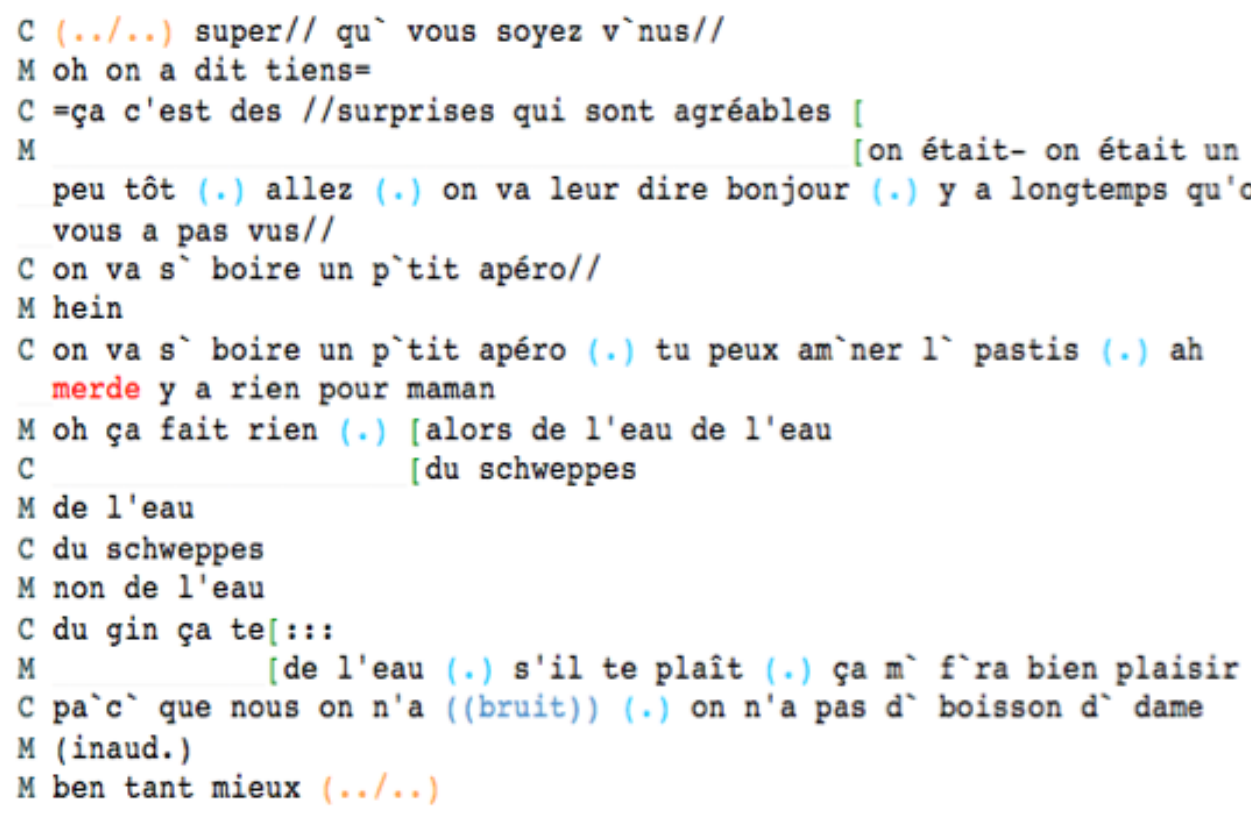

\subsection{L'émotion émergente comme syndrome}

\subsubsection{L'émotion syndrome}

Les psychologues considèrent l'émotion comme un syndrome affectant un individu. Un syndrome est un phénomène complexe, un ensemble intégré de composantes entre lesquelles les relations sont problématiques. Le mot est emprunté au vocabulaire médical, où il désigne un ensemble de symptômes (signes) sans cause spécifique, que le malade est susceptible d'avoir en même temps lors de certaines maladies. Exemple: le syndrome parkinsonien associe une akinésie (les mouvements sont lents et rares), un tremblement pendant le repos et une hypertonie (les muscles de l'organisme présentent des contractions inappropriées) [14]. La maladie est une transformation de la personne, mais toutes les transformations de la personne ne sont pas des maladies.

Selon $[15,16]$, l'émotion s'analyse selon cinq composantes : (i) évaluation cognitive des situations ; (ii) activation psychologique; (iii) expression motrice ; (iv) comportement et ébauche d'action; (v) sentiment subjectif.

\subsubsection{Structure sémantique des termes d'émotion}


L'analyse psychologique de l'émotion comme syndrome à plusieurs composantes correspond à l'analyse sémantique du mot émotion (considéré comme un concept) au moyen de traits sémantiques correspondant à ces composantes :

Mental conditions always have either a significant Cognitive component or a significant Affective component, and sometimes both. In addition, some have a significant Behavioral component. It may be that truly psychological conditions generally implicate all of these facets to some degree. However, many of the words in the affective lexicon, while of course having affective overtones, do not have affect as a significant part of their referential focus [...] [W] hen, for example, we claim that "proud" has an affect as a significant component, we do not mean to deny that it has a cognitive component and (possibly) even a behavioral one. [souligné par nos soins] [17, p. 351-352]

Le contenu sémantique des termes d'émotion peut s'analyser selon ces trois dimensions de l'émotion [17]. On remarque que cooperative et opimistic reçoivent deux focus.

Table 1. The referential structure of the affective lexicon [17]

\begin{tabular}{|l|c|c|c|}
\hline word & Focus on affect & Focus on cognition & Focus on behaviour \\
\hline proud & + & & \\
\hline confused & & + & \\
\hline cooperative & & + & + \\
\hline glad & + & + & \\
\hline optimistic & + & + & \\
\hline
\end{tabular}

Nous utiliserons une version légèrement modifiée de cette grille sémantique :

— Le trait "behaviour" est défini comme visant les (ébauches d')actions de l'expérienceur.

- Le trait complexe VMPG (verbal, mimique, postural, gestuel) est introduit comme un quatrième trait, pour souligner le fait que l'émotion est une expérience corporelle. Ce composant est essentiel lorsqu'il s'agit d'opérer des inférences émotionnelles à partir de la description de l'expérienceur.

- On peut également introduire un trait contrôle, qui distingue peur de panique.

Cette grille sémantique peut également être appliquée aux termes couvrants $(\S 1)$.

\subsection{L'émotion émergeante est une vague}

D'un point de vue interne individuel comme du point de vue interactif, l'émotion peut être caractérisée comme un phénomène phasique. En médecine, un phénomène est dit phasique lorsqu'il se développe rapidement et fortement sous l'action d'un stimulus, puis s'adapte rapidement après une courte période d'excitation [18].

Les émotions émergentes peuvent être considérées comme des phénomènes phasiques consistant en une variation de l'excitation suivie d'une récupération d'un état d'excitation standard. L'épisode émotionnel peut être représenté grossièrement comme une fluctuation phasique de premier plan, par rapport à une ligne stable d'arrière-plan. Cette ligne de fond correspond au degré d'excitation routinier de l'expérienceur, engagé dans telle ou telle action, dans des circonstances données. Ce développement définit un épisode émotionnel. 


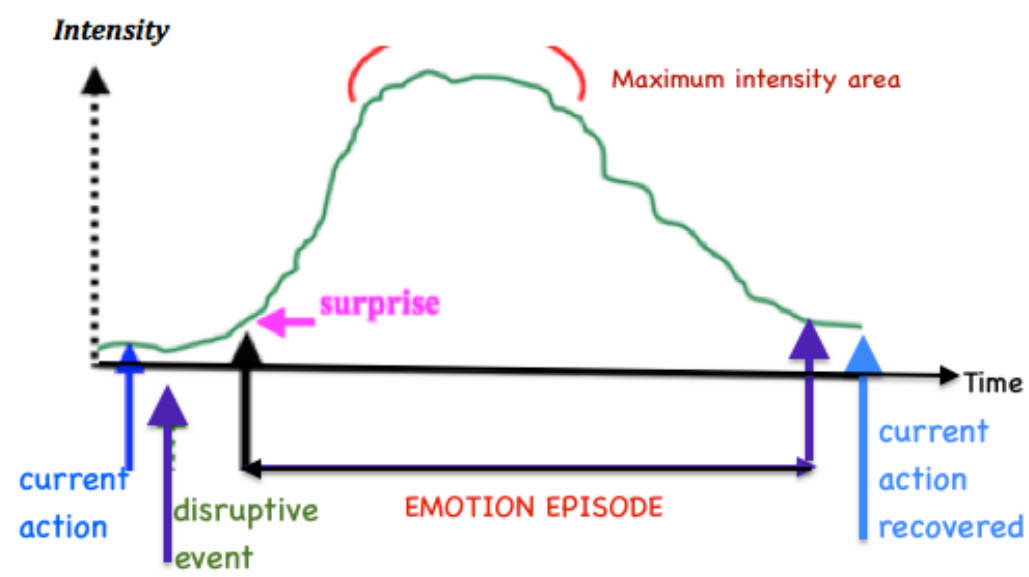

Fig. 1. Episode émotionnel

Cette représentation de l'épisode émotionnel sous la forme d'une vague est empiriquement adéquate et très pratique. Elle est particulièrement bien adaptée au cas de micro-émotions en interaction, comme en témoignent les interjections (voir §4.2.2). Elle peut néanmoins être critiquée, car elle implique que quelque chose comme le degré zéro d'excitation, autrement dit le calme, est l'état normal de la personne en interaction avec ses partenaires et avec son milieu, "the normal state of composure". Cela suggère à tort que l'émotion est quelque chose d'exceptionnel et d'anormal, une maladie de l'âme opposée au cours sain de la vie mentale ordinaire et au déroulement sans problème de l'action courante.

De plus, une telle représentation postule à tort deux types de symétrie : d'une part, une symétrie entre le temps pré-émotionnel (action en cours) et le temps post-émotionnel (récupération de l'action en cours, comme si de rien n'était), ce qui n'est pas forcément le cas (certaines émotions cassent l'action en cours) ; d'autre part, la courbe émotionnelle ellemême n'est pas symétrique, la phase d'émergence de l'émotion (courbe montante) peut être très brève, alors que la phase de contrôle (résorption et réélaboration) peut être très longue.

\subsubsection{La vie émotionnelle comme succession de vagues}

La vie interactionnelle quotidienne ne doit pas être représentée comme un état anémotionnel calme et durable, perturbé par quelques émotions rares et bien caractérisées, mais comme une série d'impulsions émotionnelles successives, continues et coordonnées.

Dans les interactions, le cumul d'événements interactionnels tels que les chevauchements, l'intensité de la voix, le flux lent / rapide de la parole peut être considéré comme un critère caractérisant les moments émotionnels. Cela ouvre la voie à une détection automatique des épisodes d'émotion dans les interactions.

La ligne ondulée qui en résulte combine de petites émotions à des émotions plus grandes, représente le "parcours émotionnel" des expérienceurs sous la forme d'une succession d'épisodes de tension / détente [19]. 


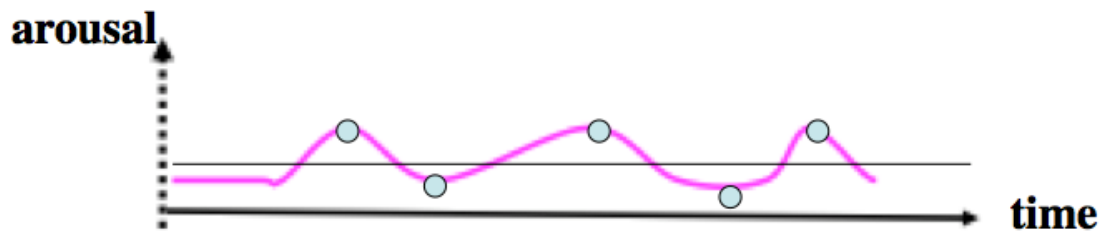

Fig. 2 Parcours émotionnel

La figure 1 représente les émotions sous la forme d'une courbe, émergeant d'une ligne droite non émotionnelle. Cette ligne droite peut être considérée comme représentant les attitudes de routine attendues dans un groupe donné, au cours d'une activité donnée.

La figure 2 ne postule pas une telle ligne droite. Néanmoins, une ligne de tension moyenne peut être déterminée en coupant les moments saillants. La différence avec la figure 1 est que la ligne ne postule nullement l'existence d'états non émotionnels, mais qu'elle représente le niveau moyen de tension dans une succession d'événements coordonnée.

Le "normal state of composure" apparaît maintenant comme une construction et non comme l'état mental fondamental de l'individu.

\subsection{Récapitulation : l'émotion comme expérience}

L'émotion est une expérience (au sens de "faire l'expérience de -"). Cette expérience affecte, est menée par un expérienceur; elle est relativement consciente, plus ou moins agréable ou désagréable. Elle est temporellement limitée (phasique, séquentielle). Elle se déroule sur un état de fond thymique (thymique = qui concerne l'humeur, le tempérament de l'expérienceur, voir §2).

L'expérience émotionnelle est située; elle est liée à la perception d'une situation qui mobilise les ressources cognitives et les valeurs de l'expérienceur. Le système cognitif opère en amont (analyse - évaluation de la situation) et en aval (gestion de l'émotion). La gestion, éventuellement contrôlée, du décours émotionnel fait partie de l'épisode émotionnel.

Cette expérience est caractérisée par une modification de l'éthos corporel de l'expérienceur : voix, mimiques, postures, gestes. Ces modifications VMPG constituent un système sémiotique par lequel se construit et se communique l'émotion.

En résumé, l'émotion est une modalité spécifique, structurée, de la communication, de l'action et de l'interaction.

\section{Rôles clés et cadre linguistique de l'expression- communication émotionnelle}

\subsection{Cadres}

L'expression émotionnelle peut être appréhendée à l'aide de deux schématisations, un cadre linguistique de base et un cadre étendu.

\subsubsection{Cadre linguistique de base}

Le cadre linguistique de base de l'expression émotionnelle est défini par la relation entre un expérienceur (Exp), une émotion (Ém) et une situation source (S) :

Qui ? Ressent quoi ? Pourquoi?

Expérienceur - Émotion - Situation 


\section{- Quoi ? L'émotion ou zone d'affect}

Première étape : repérer dans le corpus les termes relatifs à l'émotion. La présence d'un terme d'émotion n'est pas un critère nécessaire pour caractériser un texte écrit ou oral comme émotionnel. Les émotions peuvent être inférées de mots principalement descriptifs, soit description de la situation, de l'expérienceur, ou de la tension de l'énonciation, voir [20].

Deuxième étape : repérer dans le corpus les termes ayant une orientation émotionnelle. On trouve de tels termes dans les éléments descriptifs 1) de la situation ; 2) des expressions faciales, corporelles et gestuelles de l'expérienceur ; 3) de la nouvelle orientation prise par les activités de ce dernier. L'inférence d'un terme non émotionnel à une émotion est légitime si elle est fondée sur la structure sémantique du lexique, telle qu'elle est enregistrée dans les dictionnaires. Les extrapolations fondées sur la simple intuition de l'analyste risquent d'être arbitraires et artificielles.

\section{- Qui ? L'expérienceur}

Les émotions dites étant repérées, il faut ensuite attacher ces émotions à leurs expérienceurs. La tâche peut sembler facile, mais ne l'est pas ; voir le cas de l'énoncé “c'est un enfant abandonné" [17].

\section{- Pourquoi? La cohérence situation-émotion}

Les émotions ont leur source dans une situation vue (décrite) dans la perspective d'un expérienceur spécifique (voir règles et exemples dans [12]).

\subsubsection{Cadre étendu}

Pour rendre compte de la complexité des données, le cadre de base précédent doit être complété par deux autres acteurs clés de la scène émotionnelle, l'allocateur et, dans les textes écrits, l'orchestrateur des émotions et des affects.

L'allocateur attribue des émotions aux expérienceurs :

Qui dit que [quelqu'un - ressent telle émotion — dans telle situation] ?

Allocateur [Expérienceur, Émotion, Source]

L'orchestrateur répartit les émotions sur un ensemble d'expérienceurs via différents allocateurs :

Qui dit que \{tel allocateur dit que [quelqu'un ressent telle émotion dans telle situation]\}?

$$
\text { Orchestrateur }\left\{\begin{array}{l}
\text { Allocateur1 (Exp1, Ém1, Source1) } \\
\text { Allocateur2 (Exp2, Ém2, Source2) }
\end{array}\right.
$$

\subsection{Rôles émotionnels : Expérienceur, Allocateur, Orchestrateur}

L'expérienceur est l'être qui vit l'expérience émotionnelle. Qui peut être un expérienceur? Les humains, les animaux supérieurs, les végétaux ? On en décide en fonction des capacités de conscience de l'être considéré et de ses capacités à éprouver du plaisir / déplaisir. Les inanimés ne sont pas les expérienceurs des émotions qui peuvent leur être attachées, un paysage mélancolique.

On distingue les expérienceurs actuels et les expérienceurs potentiels. Considérons le report narratif d'un événement impliquant différents personnages. Tous ces personnages sont des expérienceurs potentiels. Néanmoins, le locuteur-orchestrateur peut attribuer des 
émotions uniquement à certains d'entre eux, les expérienceurs actuels. C'est un fait essentiel à observer lors de la description de l'émotion dans des situations complexes.

La prise en compte du témoignage émotionnel demande que l'on distingue l'expérienceur en dernière instance (par exemple, le téléspectateur) et l'expérienceur relai, la personne qui lui transmets l'information émotionnelle, le présentateur du journal télévisé par exemple. Dans le cas du témoin interviewé faisant part de son émotion à un interviewer lui-même cadré par un présentateur, trois relais interviennent dans la transmission de l'émotion jusqu'à sa cible, l'expérienceur en dernière instance, le téléspectateur. L'alignement parfait des expérienceurs relais est un facteur essentiel dans la création d'un consensus émotionnel, produisant l'illusion de la naturalité de l'émotion.

L'allocateur attribue une émotion à un expérienceur. L'émotion est auto-attribuée quand l'expérienceur lui-même dit qu'il est dans tel ou tel état émotionnel : J'ai peur.

L'émotion est hétéro-attribuée quand une autre personne que l'expérienceur, l'allocateur, dit que l'expérienceur est dans tel ou tel état émotionnel : Pierre a peur.

Par exemple, dans un roman comme dans la narration quotidienne d'un événement émotionnel, des émotions sont attachées aux personnes 1) directement, par le locuteurnarrateur, Pierre est triste, ou 2) indirectement par d'autres participants, Paul dit que Pierre est triste. Dans les deux cas, le locuteur-narrateur orchestre l'allocation des émotions sur les personnages impliqués dans la narration.

L'orchestrateur met en scène un événement émotionnel et distribue aux participants des émotions selon sa perspective narrative, notamment via un système d'allocateurs. Dans une interaction, le rôle d'orchestrateur n'est pas préétabli ; l'orchestration émerge de l'interaction elle-même.

\subsection{Exercice}

Dans le texte suivant, Rosalía de Castro, une poétesse profonde et inspirée, décrit son pays, la Galice ; le texte original est en galicien. Il permet de récapituler les différents cadres et rôles émotionnels.

Lacs, cascades, torrents, vallées fleuries, gorges, montagnes, cieux bleus et calmes comme ceux de I'Italie, horizons nuageux et mélancoliques, mais toujours beaux comme ceux, si vantés, de la Suisse ; rivages paisibles, caps tempétueux terrifiants et admirables dans leur sourde et grandiose colère... mers immenses... que dire de plus? Aucune plume ne peut décrire autant d'enchantements. Une terre couverte d'herbe et de fleurs en toutes saisons; des montagnes couvertes de pins, de chênes et de saules ; des brises caressantes ; des fontaines et des ruisseaux qui répandent leurs eaux cristallines et babillantes, été comme hiver, à travers des champs joyeux ou des gorges sombres et profondes... La Galice est un jardin où on respire parfums, fraîcheur et poésie... Et pourtant, l'arrogance des ignorants est si extrême, leurs préjugés indécents contre notre terre sont tellement enracinés que ceux-là mêmes qui ont contemplé de telles splendeurs (sans parler de ceux qui se moquent de nous sans nous avoir jamais vu de près ni de loin, et ils sont les plus nombreux), même ceux qui ont visité la Galice et apprécié ses délices, ont osé dire que la Galice était... une porcherie répugnante !! [un cortello inmundo!!]

Rosalía de Castro, Cantares Gallegos (1863). Édition de María Xesús Lama López (1995). Vigo, Galaxia. 


\section{Conclusion: Construire une représentation d'un épisode émotionnel}

La parole émotionnée est une forme de langage et d'interaction que l'on identifie intuitivement. Son analyse procède à partir de données, corpus ou échantillons, que l'on estime particulièrement pertinentes, c'est-à-dire susceptibles de livrer des résultats intéressants du point de vue linguistique et interactionnel. La tâche analytique s'appuie sur des savoirs, se développe de façon méthodique, et se concrétise en une représentation des données, c'est-à-dire un discours à propos de ces données.

Cette tâche doit nécessairement aborder, à un moment ou à un autre, la question des termes d'émotion. Dans la première partie ( $\$ 2$ et 3 ), nous avons montré que, loin de se limiter à quelques substantifs ou dizaines de substantifs, le lexique de l'émotion touche à des centaines, voire des milliers de termes, liés à l'un ou l'autre aspect du développement séquentiel de l'émotion. Cette abondance fournit à l'analyse une base d'autant plus sûre qu'elle s'appuie sur des jugements qui ne font pas appel à l'intuition subjective de l'analyse, les définitions des dictionnaires.

L'étude lexicale de l'émotion considère le mot, simple ou composé ; l'étude syntaxique, l'énoncé construit autour d'un verbe psychologique ; l'étude de l'émotion dans la parole prend pour objet propre la séquence émotionnelle, composée d'une série d'énoncés ou de tours de parole coordonnés. Les critères qui permettent de découper cette séquence dans un flux de parole sont les critères classiques de cohérence sémantique interne et de changement de thèmes ou d'actions aux frontières.

Les séquences émotionnelles sont de deux types principaux (§4). La séquence émotionnelle primaire est constituée par l'émotion émergente. Cette émotion peut se résorber au fil de la parole, comme dans le cas des micro-émotions de la vie quotidienne, telles qu'elles se manifestent autour d'une exclamation. Les grandes émotions sont caractérisées par leur reprise dans des séquences émotionnelles secondaires, qui réactivent l'émotion primaire, la racontent, l'élaborent et/ou la contrôlent, intégrant l'émotion à la vie ordinaire ou réaménageant la vie ordinaire autour des perspectives ouvertes par une expérience émotionnelle jugée décisive.

Quel que soit l'intérêt qui pousse l'analyste vers les situations émotionnelles et leur langage, l'approche linguistique de l'émotion suppose une certaine mise à distance de l'empathie. L'empathie est un puissant instrument de compréhension intuitive de l'émotion, mais, à s'y livrer sans contrôle, l'analyste risque de s'engluer dans des données qu'il ne fait que reformuler.

Le recours à des cadres généraux de l'émotion permet une certaine objectivation de l'analyse (§5), et livre des résultats féconds et parfois inattendus. La séquence émotionnelle s'organise autour du cadre fondamental Expérienceur - Émotion - Situation; il s'ensuit que les premières questions analytiques doivent porter sur les éléments de ce cadre : dans la situation émotionnelle étudiée, QUI ? éprouve QUOI? Et POURQUOI? Ces questions sont elles-mêmes prises dans le jeu des attributions d'émotion par les allocateurs d'émotion : QUI dit que (tel expérienceur éprouve telle émotion dans telle situation)? À l'écrit, l'orchestrateur des émotions attribue ou non les émotions aux expérienceurs, directement ou via les allocateurs dont il dispose.

Pour des raisons d'espace, nous ne pouvons pas discuter ici le rôle de la situation en relation avec l'émotion. L'émotion n'est pas liée mécaniquement à une situation comme le stimulus peut l'être à une réponse, comme on le voit immédiatement si l'on considère que tous les participants à une même situation sont loin d'éprouver la même émotion, ou d'ailleurs d'éprouver quoi que ce soit. L'émotion est liée à une description de la situation; c'est ce qui rend l'émotion accessible au langage. Cette description apparaît lorsqu'il s'agit de justifier l'émotion; des conflits d'émotions se traduisent par des conflits de description, 
et réciproquement. La position émotionnelle se déduit de la description selon les règles de l'inférence émotionnelle; elle s'argumente comme n'importe quel point de vue.

Pour cette même raison de place, nous ne pouvons pas aborder la question des signes émotionnels. L'émotion est décelable comme signe naturel (variation de la conductivité de la peau) dans la mesure où son substrat neurophysiologique se manifeste dans ou sur le corps de l'expérienceur. Elle se manifeste également comme signe conventionnel, entrant dans un système spécifique de communication et d'interaction. En premier lieu, l'émotion est dite et communiquée comme peut l'être n'importe quel phénomène, selon les ressources d'un système linguistique, dont le support est l'activité des cordes vocales. En second lieu, l'émotion est dite et communiquée selon les ressources d'un système sémiotique spécifique, fondé matériellement sur l'activation de tout le corps de l'expérienceur : sur-activation des cordes vocales (modification de la voix parlée), des mimiques faciales, des postures du corps et des gestes.

L'étude de cette production sémiotique de l'émotion doit être menée différemment selon le type de données considérées. S'il s'agit de prises vidéo d'interactions authentiques, l'étude nécessite des compétences spécialisées multiples. Pour les interactions mises en scène, vidéo ou image, l'étude relève d'une compétence sémiotique émotionnelle, telle qu'elle est définie dans la culture concernée. L'inférence émotionnelle à partir de tel trait peut légitimement s'appuyer sur l'intuition sémiotique, comme la compréhension d'un énoncé s'appuie sur l'intuition linguistique; dans les deux cas, s'il y a doute, l'intuition peut être contrôlée par le recours à la méthode du consensus. Enfin, à l'écrit, le système sémiotique émotionnel est verbalisé dans une description du comportement de l'expérienceur. À partir de telle ou telle expression décrivant ce comportement, telle ou telle émotion est attribuée à l'expérienceur. L'inférence est justifiée par la méthode des impacts présentée dans les trois premières sections de cet article.

\section{Bibliographie}

1. É. Coquery \& A. Piéjus, Figures de la passion (Musée de la Musique, Paris, 2001)

2. É. Coquery \& A. Piéjus, L'invention du sentiment (Musée de la Musique, Paris, 2002)

3. Chr. Plantin, Emotion and Affect. Dans K. Tracy, C. Ilie \& T. Sandel (éds), The International Encyclopedia of Language and Social Interaction, 514-523 (John Wiley \& Sons, New York, 2015),

4. Aristote, Éthique à Nicomaque (Éditions Les Échos du Maquis, 1959).

5. D. Galati, B. Sini, Les structures sémantiques du lexique français des émotions. Dans Chr. Plantin, M. Doury, V. Traverso (éds), Les émotions dans les interactions, 75-87 (PUL, Lyon, 2000)

6. http://www.synonymo.fr/synonyme/abasourdi

7. https://cnrtl.fr/definition/col\%C3\%A8re

8. Chr. Plantin, Dissémination de colère dans le lexique français : Un exercice sur les termes et les expressions d'émotion. Myriades 3, 1-24 (2017).

9. Chr. Plantin, La dissémination de émotion dans le lexique. Dans A. Krzyżanowska, K. Wołowska (éds), Les émotions et les valeurs dans la communication, 109-133 (Peter Lang, Berne, 2016)

10. P. Blumenthal, Particularités combinatoires du français en Afrique: essai méthodologique. Le français en Afrique 27, 55-74 (2012). 
11. R. Bouchard, M'enfin !!! Des petits mots pour les petites émotions ? Dans Chr. Plantin, M. Doury, V. Traverso (éds), Les émotions dans les interactions, 223-238 (PUL, Lyon, 2000)

12. Chr. Plantin, Publier son émotion - et partir sans payer (241-252);-Dites, y'avait une agrafe : copiloter des émotions (253-277). Dans Les bonnes raisons des émotions. Principes et méthodes pour l'étude de la parole émotionnée (Peter Lang, Berne, 2011).

13. Chr. Plantin, Micro-émotions en interaction : Ah merde, y a rien pour maman. Voix Plurielles 12, 1 (2015).

14. https://www.vulgaris-medical.com/encyclopedie-medicale/syndrome

15. K. R. Scherer, Les émotions: Fonctions et composantes. Cahiers de psychologie cognitive 4, 9-39 (1989)

16. K. R. Scherer, On the nature and function of emotion: A component process approach. Dans K. R. Scherer, P. Ekman (éds), Approaches to emotion, 293-317 (Lawrence Erlbaum, Hillsdale, 1984)

17. A. Ortony, G. L. Clore, M. A. Foss, The referential structure of the affective lexicon, Cognitive Science, 11, 341-364 (1987)

18. https://www.merriam-webster.com/medical/phasic

19. M. Quignard, B. Ursi, N. Rossi-Gensane, V. André, H. Baldauf-Quilliatre, C. Etienne, Chr. Plantin, V. Traverso. Une méthode instrumentée pour l'analyse multidimensionnelle des tonalités émotionnelles dans l'interaction. SHS Web of Conferences $27: 15004$ (2016).

20. Chr. Plantin, Tense arguments: Questions, exclamations, emotions. Informal Logic 39, 347-371 (2019). 